\title{
Co-culture of fibroblast-like synoviocytes with umbilical cord-mesenchymal stem cells inhibits expression of pro-inflammatory proteins, induces apoptosis and promotes chondrogenesis
}

\author{
JINGQI ZENG ${ }^{1}$, FAN WANG $^{1}$ and MINZHI MAO ${ }^{2}$ \\ ${ }^{1}$ Department of Orthopedics, The Second Affiliated Hospital of Hunan University of Chinese Medicine, \\ Changsha, Hunan $410005 ;{ }^{2}$ Department of Orthopedics, \\ The Second Xiangya Hospital of Central South University, Changsha, Hunan 410011, P.R. China
}

Received July 24, 2015; Accepted August 9, 2016

DOI: $10.3892 / \mathrm{mmr} .2016 .5721$

\begin{abstract}
The present study aimed to investigate the effect of co-culture of fibroblast-like synoviocytes (FLS) with human umbilical cord-mesenchymal stem cells (UC-MSCs) on rheumatoid arthritis (RA) and to understand the mechanisms that mediate the induced changes. FLS and UC-MSCs were isolated and cultured individually, FLS were then cultured with or without UC-MSCs. The phenotype of UC-MSCs was analyzed prior to co-culture. The UC-MSCs were successfully isolated and expanded, and exhibited a fibroblast-like morphology. Enzyme-linked immunosorbent assay (ELISA) and reverse transcription-quantitative polymerase chain reaction (RT-qPCR) were performed to determine the expression levels of interleukin (IL)-1 $\beta$, IL-6, and chemokine (C-C motif) ligand (CCL)-2. The cell apoptosis rate was determined by flow cytometry. Furthermore, the RNAs of aggrecan and collagen type II were isolated and assessed in a chondrogenesis assay following co-culture for 7, 14, 21 and 28 days. Protein expression levels of apoptosis-related proteins, including B-cell lymphoma (Bcl-2), Bcl-2-associated X protein, p53 and phospho (p)-AKT, and growth differentiation factor-5 were analyzed by western blotting. ELISA and qRT-PCR demonstrated that compared with FLS cultured alone, co-culture with UC-MSCs significantly downregulates the expression levels of IL-1 $\beta$, IL-6 and CCL-2. Additionally, the percentage of apoptotic cells was significantly increased in the co-cultured cells $(\mathrm{P}<0.05)$, and the relative RNAs levels of aggrecan and collagen type II were increased compared with FLS alone.
\end{abstract}

Correspondence to: Dr Minzhi Mao, Department of Orthopedics, The Second Xiangya Hospital of Central South University, 139 Renmin Road, Changsha, Hunan 410011, P.R. China

E-mail: maominzhi973@126.com

Key words: rheumatoid arthritis, fibroblast-like synoviocytes, umbilical cord mesenchymal stem cells, co-culture
Furthermore, the expression levels of Bcl-2 $(\mathrm{P}<0.05)$ and p-AKT $(\mathrm{P}<0.05)$ were significantly decreased, whereas, $\mathrm{p} 53$ $(\mathrm{P}=0.001)$, Bax $(\mathrm{P}<0.01)$ and GDF-5 $(\mathrm{P}<0.01)$ were increased by co-culture of FLS with UC-MSCs compared with FLS alone. In conclusion, co-culture of FLS with UC-MSCs may be important and clinically useful for the treatment of RA by inhibiting the expression of pro-inflammatory mediators, inducing apoptosis and promoting chondrogenesis.

\section{Introduction}

Rheumatoid arthritis (RA), a chronic multisystem inflammatory autoimmune disorder, is characterized by destructive synovitis, systemic inflammation and acceleration of atherosclerosis $(1,2)$. The imbalance of inflammatory cytokines, including tumor necrosis factor (TNF) and interleukin (IL)-6, is responsible for this disease (3). It has been previously estimated that $\sim 0.5 \%-1.0 \%$ of the general population are affected by RA (4). The prevalence of RA in China is $0.28 \%$, with a $6: 1$ female:male ratio (5). RA leads to various functional disabilities, pain and low health-associated quality of life, imposing a large economic and mental burden on society and individuals with the disease (6,7). The predominant selective and effective therapeutic options for RA include conventional-synthesized disease-modifying antirheumatic drugs (DMARDs) and biological DMARDs, for example, anti-TNF- $\alpha$ monoclonal antibodies and tocilizumab. Although the management of RA has achieved considerable success, various approaches are expensive and no DMARD achieves long-term drug-free remission $(8,9)$. Thus, it is necessary to develop novel and more effective therapies for RA.

Previous studies have investigated mesenchymal stem cells (MSCs) due to their immunosuppressive ability via regulation of $\mathrm{T}$ and $\mathrm{B}$ cell proliferation and differentiation (10). MSCs are considered as attractive therapeutic targets for treatment of immune disorders, including RA (11-13). The major common sources of MSCs are bone marrow, peripheral blood and adipose tissue. However, obtaining human MSCs is invasive, except when extracted from umbilical cord (UC), as it is a postnatal 
organ discarded following birth. Additionally, UC-MSCs have a higher proliferative potential than bone marrow-derived MSCs (BM-MSCs) $(14,15)$. Furthermore, it has previously been well demonstrated that UC-MSCs also possess immunoregulatory capabilities (15). Therefore, UC-MSCs are now regarded as an alternative source of stem cells and require further investigation (16). In a previous report, Liu et al (17) suggested that there is therapeutic potential in using human UC-MSCs for the treatment of RA. However, little information is available regarding the co-culture of fibroblast-like synoviocytes (FLS) with UC-MSCs.

The present study investigated the effect induced by co-culture of FLS with UC-MSCs and the potential mechanism by which this may affect RA. The levels of certain pro-inflammatory cytokines and chemokines, aggrecan, collagen type II and apoptosis-associated proteins, and the percentage of apoptotic cells were analyzed.

\section{Materials and methods}

Isolation and phenotypic identification of human UC-MSCs. The protocol of the present study was approved by the ethics committee of The Second Affiliated Hospital of Hunan University of Chinese Medicine (Changsha, China) and informed consent was obtained from all the participants prior to the research. The methods for isolation and culture of human UC-MSCs were described a previous study (18). Fresh human umbilical cords ( $n=5$; gestational age, 39-40 weeks) were obtained from Department of Obstetrics and Gynecology of The Second Affiliated Hospital of Hunan University of Chinese Medicine following normal birth. The tissues were collected and transferred into a sterile container in $\alpha$-modified Minimum Essential Medium (Thermo Fisher Scientific, Inc., Waltham, MA, USA) supplemented with $100 \mathrm{U} / \mathrm{ml}$ penicillin and $100 \mu \mathrm{g} / \mathrm{ml}$ streptomycin (Sigma-Aldrich, St. Louis, MO, USA). Following washing with phosphate-buffered saline (PBS), the tissues were divided into $1-2 \mathrm{~mm}^{3}$ pieces, incubated with $0.075 \%$ type II collagenase (Sigma-Aldrich) and $0.125 \%$ trypsin (Thermo Fisher Scientific, Inc.) then passed through a $100-\mu \mathrm{m}$ cell strainer (BD Biosciences, San Jose, CA, USA). The cells $\left(1 \times 10^{6}\right.$ cells $\left./ \mathrm{cm}^{2}\right)$ were then transferred to a flask containing the growth medium (GM) and cultured in a humidified atmosphere with $5 \% \mathrm{CO}_{2}$ at $37^{\circ} \mathrm{C}$ for 3 to 4 days. The GM included Dulbecco's modified Eagle medium (DMEM; Sigma-Aldrich) and 10\% fetal bovine serum (FBS) (Gibco; Thermo Fisher Scientific, Inc.) supplemented with $10 \mathrm{ng} / \mathrm{ml}$ vascular endothelial growth factor (VEGF; Gibco; Thermo Fisher Scientific, Inc.), $10 \mathrm{ng} / \mathrm{ml}$ epidermal growth factor (EGF; Gibco; Thermo Fisher Scientific, Inc.), $100 \mathrm{U} / \mathrm{ml}$ penicillin, $100 \mu \mathrm{g} / \mathrm{ml}$ streptomycin, and $2 \mathrm{mM}$ L-glutamine (Sigma-Aldrich). Following culture, the medium was changed and non-adherent cells were discarded. The medium was replaced twice weekly. The adherent cells $\left(1 \times 10^{4}\right.$ cells $\left./ \mathrm{cm}^{2}\right)$ were maintained in GM for expansion when the confluence reached $60-80 \%$. For phenotypic identification, the cells were detached and washed with PBS supplemented with $0.5 \%$ bovine serum albumin (BSA; Sigma-Aldrich), and maintained in primary antibodies for $30 \mathrm{~min}$ at $4^{\circ} \mathrm{C}$. To examine intracellular antigens, the cells were fixed with $4 \%$ paraformaldehyde for $15 \mathrm{~min}$ at $4^{\circ} \mathrm{C}$, blocked with normal goat serum, and then permeabilized with $0.1 \%$ saponin (Sigma-Aldrich) for $1 \mathrm{~h}$ at room temperature. Primary antibodies were used as follows: cluster of differentiation (CD)105, CD29, CD44, CD166, CD14, CD34, and CD45 (BD Biosciences). An antibody of the same isotope from the same species without a target served as the negative control. The cells were then washed with PBS containing $0.5 \% \mathrm{BSA}$ and incubated with fluorescein isothiocyanate (FITC) and phycoerythrin (PE)-conjugated secondary antibodies for $30 \mathrm{~min}$ at $4^{\circ} \mathrm{C}$. Following washing three times, the cells were resuspended in PBS and detected by flow cytometry by using FACSCalibur flow cytometer (BD Biosciences) and the CellQuest Pro 3.0 software (BD Biosciences) (19).

Isolation of FLS and co-culture with UC-MSCs. The FLS were prepared from synovial tissues of 6 patients with RA who had undergone total joint replacement surgery. The diagnosis of RA was based on the revised criteria from the American College of Rheumatology (20). The FLS were isolated according to a previously described method (21). Briefly, the tissues were washed with PBS and homogenized, then treated with type I collagenase (1 mg/ml; Sigma-Aldrich) and $2 \mathrm{mg} / \mathrm{ml}$ testicular hyaluronidase (Sigma-Aldrich) in DMEM for $2 \mathrm{~h}$ at $37^{\circ} \mathrm{C}$. Then, the culture was washed with PBS and maintained overnight in DMEM supplemented with $10 \%$ FBS, $100 \mathrm{U} / \mathrm{ml}$ penicillin, $100 \mu \mathrm{g} / \mathrm{ml}$ streptomycin and $2 \mathrm{mM}$ L-glutamine in a $5 \% \mathrm{CO}_{2}$ incubator. FLS at passages 4-6 were used for each experiment.

For the co-culture, FLS $\left(1.5 \times 10^{4}\right)$ were seeded in 24-well plates and cultured overnight in DMEM containing 10\% FBS. FLS were then washed with serum-free DMEM and UC-MSC suspensions were added directly onto the FLS at a cell number ratio of $1: 1$ for $24 \mathrm{~h}$.

Enzyme-linked immunosorbent assay (ELISA). Following co-culture, the supernatants from each group (FLS alone or FLS + UC-MSCs) were collected and centrifuged to remove cellular debris. The cell-free culture supernatants were assayed for IL-1 $\beta$, IL-6 and chemokine (C-C motif) ligand (CCL)-2 using commercial ELISA kits (R\&D Systems, Inc., Minneapolis, MN, USA). All samples and standards were assessed in triplicate. The absorbance of each well was determined at $450 \mathrm{~nm}$ with a spectrophotometer (SpectraMax 250, Molecular Devices, LLC, Sunnyvale, CA, USA). The reference wavelength was $540 \mathrm{~nm}$.

Reverse transcription-quantitative polymerase chain reaction $(R T-q P C R)$. Total RNA in the FLS alone or FLS + UC-MSCs groups was isolated using RNeasy Mini kit (Qiagen, Inc., Valencia, CA, USA) according to the manufacturer's instructions. Total RNA was quantified and complementary DNA (cDNA) was synthesized using SuperScript Reverse Transcriptase (Promega Corporation, Madison, WI, USA) following the manufacturer's recommendations. The cDNA was subsequently used for qPCR. The IL-1 $\beta$, IL-6, and chemokine CCL-2 mRNA levels were determined using PrimeScript RT Reagent kit (Takara Bio, Inc., Otsu, Japan) and SYBR Premix Ex Taq (Takara Bio, Inc.). The qPCR cycling conditions were as follows: $95^{\circ} \mathrm{C}$ for $10 \mathrm{~min} ; 40$ cycles at $95^{\circ} \mathrm{C}$ for $15 \mathrm{sec}, 60^{\circ} \mathrm{C}$ for $2 \mathrm{~min}, 70^{\circ} \mathrm{C}$ for $2 \mathrm{~min}$, and $77^{\circ} \mathrm{C}$ for $30 \mathrm{sec}, 99.9^{\circ} \mathrm{C}$ for $10 \mathrm{~min}$; the reaction was stopped at $4^{\circ} \mathrm{C}$. The following primers were used: Forward, 5'-GTCATGACTTGCCCTCAGCA-3' and reverse, 5'-GCAGGTGACCCAGTTTTTGG-3' for IL-1 $\beta$; 
forward, 5'-AAATTCGGTACATCCTCGACGGCA-3 and reverse, 5'-AGTGCCTCTTTGCTGCTTTCACAC-3' for IL-6; forward, 5'-CGCTCAGCCAGATGCAATCAAT-3' and reverse, 5'-GCTGGTGATTCTTCTATAGCTCGCG-3' for CCL-2; and forward, 5'-CCAAGGTCATCCATGACAAC-3' and reverse, 5'-TGTCATACCAGGAAATGAGC-3' for GAPDH. The relative expression levels of the genes were calculated by using the $2^{-\Delta \Delta \mathrm{Cq}}$ method (22). GAPDH was used as a reference gene. The reactions were performed in triplicate.

Flow cytometry assay. Cell apoptosis rate was determined by an Annexin V-FITC apoptosis detection kit (Sigma-Aldrich) according to the manufacturer's protocol. Briefly, cells were harvested and washed with cold PBS 3 times. The cells were then resuspended in $0.5 \mathrm{ml}$ binding buffer, and incubated with $10 \mu \mathrm{l}$ Annexin V-FITC and $10 \mu \mathrm{l}$ propidium iodide at room temperature in the dark for $15 \mathrm{~min}$. The cells were subsequently analyzed using a FACSCalibur flow cytometer. The percentage of total apoptotic cells, including the early and late stage, were calculated. The data were analyzed using CellQuest Pro 3.0 software.

Chondrogenesis assay. For chondrogenic differentiation, FLS with or without UC-MSCs $\left(1 \times 10^{6}\right)$ were seeded in 24-well plates with chondrogenic basal medium and cultured for 28 days at $37^{\circ} \mathrm{C}$ with $5 \% \mathrm{CO}_{2}$ and $95 \%$ air. The medium was replaced every 3-4 days. Control cells were cultured in medium without growth factors. Cells were harvested and the mRNA expression of aggrecan and collagen type II was measured following co-culture at 7, 14, 21 and 28 days using NucleoSpin RNAII kit (Machery-Nagel GmbH, Düren, Germany). cDNA was prepared by reverse transcription of total RNA. The primer sequences for aggrecan, collagen type II and the housekeeping gene were listed as follows: Forward, 5'-AGG AGACAGAGGGACACGTC-3' and reverse, 5'-TCCACTGGT AGTCTTGGGCAT-3' for aggrecan, and forward, 5'-TTCAGC TATGGAGATGACAATC-3' and reverse, 5'-AGAGTCCTA GAGTGACTGAG-3' for collagen type II. GAPDH served as the housekeeping gene. cDNA was then quantified by qPCR using SYBR Green dye. The details of cycling conditions and the quantification method were as described above.

Western blot analysis. Western blotting was performed to determine the activation of $\mathrm{Bcl}-2$ apoptosis regulator, Bcl-2-associated $\mathrm{X}$ apoptosis regulator (Bax), p53, phosphorylation of AKT serine threonine kinase and growth differentiation factor-5 (GDF-5). Protein was exacted using radioimmunoprecipitation assay buffer (Sigma-Aldrich), and the concentration was measured with the Bio-Rad DC Protein Assay kit (Bio-Rad Laboratories, Inc., Hercules, CA, USA). Protein samples $(20 \mu \mathrm{l})$ were separated on a 10-12\% sodium dodecyl sulfate-polyacrylamide gel, transferred onto polyvinylidene difluoride membranes (Merck Millipore, Darmstadt, Germany), blocked in 5\% non-fat milk powder in PBS for $2 \mathrm{~h}$ at room temperature and probed with rabbit monoclonal anti-Bcl-2 (cat. no. 4223), rabbit monoclonal anti-Bax (cat. no. 5023), anti-p53 (cat. no. 2527), anti-phosphorylated (p)-Akt (cat. no. 4060) and anti-GDF-5 (Abcam, Cambridge, MA, USA; cat. no. ab93855) primary antibodies (diluted 1:1,000) overnight at $4^{\circ} \mathrm{C}$, followed by incubation with the appropriate goat anti-rabbit horseradish peroxidase-conjugated secondary antibodies (1:5,000; cat. no. 7071$)$ for $1 \mathrm{~h}$ at $4^{\circ} \mathrm{C}$. All antibodies, unless otherwise stated, were purchased from Cell Signaling Technology, Inc. (Danvers, MA, USA). Rabbit monoclonal GAPDH (Cell Signaling Technology, Inc.; cat. no. 5174) was used as a control. Finally, the samples were detected by enhanced chemiluminescence (Pierce ECL Western Blotting Substrate; Thermo Fisher Scientific, Inc.) and densitometry analysis was performed at least three times using ImageLab ${ }^{\mathrm{TM}}$ software version 2.0.1 (Bio-Rad Laboratories, Inc.).

Statistical analysis. The experimental data are presented as the mean \pm standard deviation. Statistical analyses were performed using SPSS statistical software (version 19.0; IBM SPSS, Armonk, NY, USA). Student's t-test was performed to comparison between 2 groups. $\mathrm{P}<0.05$ was considered to indicate a statistically significant difference.

\section{Results}

Phenotypic identification of UC-MSCs. The UC-MSCs were successfully isolated and expanded and flow cytometry was performed to determine the cell phenotype. The percentages of the positive cells were calculated They were negative for CD14

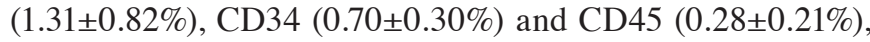
but positive for CD105 $(99.01 \pm 0.04 \%)$, CD29 $(99.23 \pm 0.31 \%)$, CD44 (98.06 $\pm 0.71 \%)$ and CD166 $(87.63 \pm 0.07 \%)$, indicating that UC-MSCs exhibited a fibroblast-like phenotype.

Effect of co-culture on expression of pro-inflammatory cytokines and chemokines. To investigate the effect of co-culture on expression levels of inflammatory cytokines and chemokines, ELISA and RT-qPCR were performed to determine the protein and mRNA levels of IL-1 $\beta$, IL- 6 and CCL- 2 . The ELISA results (Fig. 1A) demonstrated that co-culture of FLS with UC-MSCs significantly downregulated the protein expression of IL-1 $\beta(\mathrm{P}=0.000)$, IL-6 $(\mathrm{P}=0.004)$, and chemokine CCL-2 $(\mathrm{P}=0.023)$ compared with FLS cultured alone. RT-qPCR analysis demonstrated similar results. IL-1 $\beta(\mathrm{P}=0.006)$, IL-6 $(\mathrm{P}=0.036)$ and CCL-2 ( $\mathrm{P}=0.005)$ mRNA levels were significantly reduced in the co-culture group compared with the FLS only (Fig. 1B). These results indicate that co-culture of FLS with UC-MSCs inhibits the expression of pro-inflammatory cytokines and chemokines.

Effect of co-culture on FLS apoptosis and chondrogenesis. To investigate the effect of co-culture with UC-MCSs on FLS apoptosis and chondrogenesis, flow cytometry and chondrogenesis assay were performed, respectively. As demonstrated in Fig. 2A and B, the percentage of apoptotic cells was significantly higher in the co-culture group compared with the FLS group ( $\mathrm{P}=0.039)$. Additionally, the relative RNA expression levels of aggrecan and collagen type II at 7, 14, 21 and 28 days were significantly higher in the co-culture group compared with the FLS group (Fig. 3). These results demonstrated that co-culture of FLS with UC-MSCs induces FLS apoptosis and promotes chondrogenic differentiation.

Effect of co-culture on the protein expression levels of apoptosis-associated proteins and GDF-5. To further confirm 

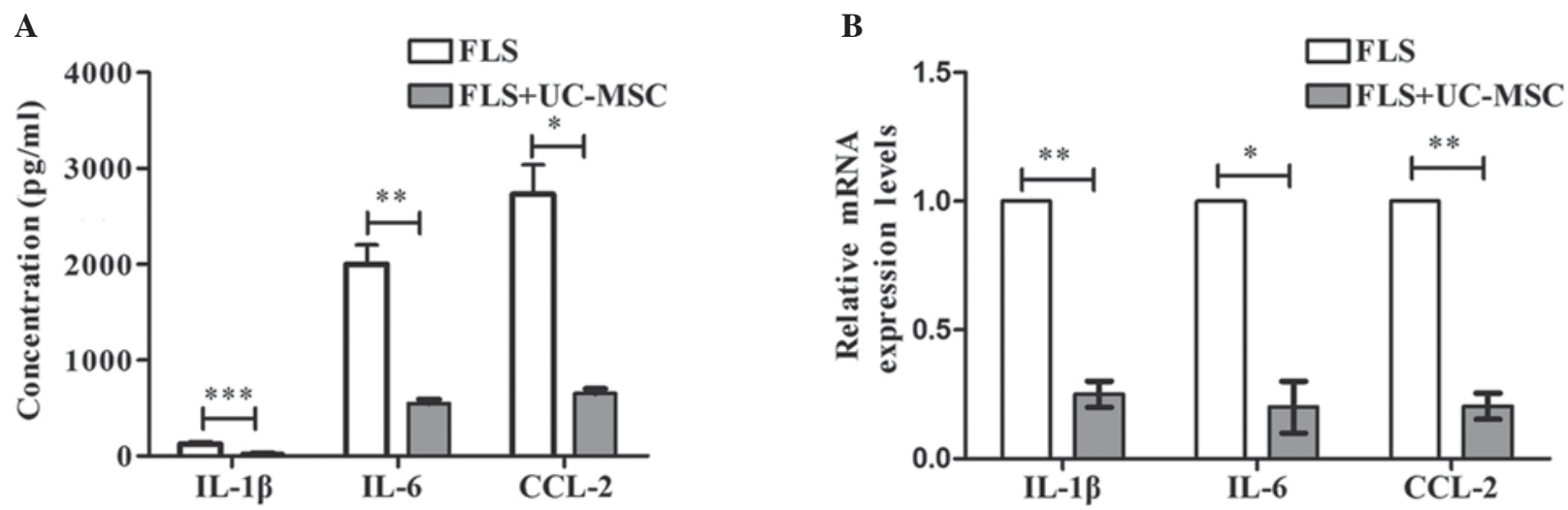

Figure 1. Effect of co-culture on expression of inflammatory cytokines and chemokines. (A) Results of enzyme-linked immunosorbent assay. (B) Results of reverse transcription-quantitative polymerase chain reaction. ${ }^{*} \mathrm{P}<0.05,{ }^{* *} \mathrm{P}<0.01,{ }^{* * *} \mathrm{P}=0.001$, comparison indicated by brackets. Data are presented as the mean \pm standard deviation. FLS, fibroblast-like synoviocyte; UC-MSC, umbilical cord mesenchymal stem cell; IL, interleukin; CCL, chemokine (C-C motif) ligand.

the mechanisms of apoptosis and chondrogenesis induced by the co-culture, the expression levels of apoptosis-associated proteins (Bcl-2, Bax, p53, and p-AKT) and GDF-5 were determined by western blot analysis. As demonstrated in Fig. 4, compared with the levels in the FLS group, the expression levels of anti-apoptotic Bcl-2 ( $\mathrm{P}=0.026)$ and $\mathrm{p}-\mathrm{AKT}(\mathrm{P}=0.019)$ were significantly decreased and the levels of pro-apoptotic p53 $(\mathrm{P}=0.001)$ and $\mathrm{Bax}(\mathrm{P}=0.006)$ were significantly increased by co-culture of FLS with UC-MSCs. Additionally, the expression level of GDF-5 was significantly increased by co-culture of FLS with UC-MSCs compared with FLS alone ( $\mathrm{P}=0.005)$. These results indicate that co-culture of FLS with UC-MSCs induces apoptosis of FLS by regulating the expression of pro/anti-apoptotic proteins.

\section{Discussion}

The present study provided evidence indicating that co-culture of FLS with UC-MSCs exerts a profound inhibitory effect on the expression of pro-inflammatory cytokines and chemokines (IL-1 $\beta$, IL-6 and CCL-2), induces FLS apoptosis and promotes chondrogenic differentiation. Furthermore, the current study demonstrated that the apoptosis and chondrogenesis induced by co-culture of FLS with UC-MSCs may be regulated via changes to the expression of pro/anti-apoptotic proteins and GDF-5.

RA is a heterogeneous autoimmune disease that not only causes progressive joint deterioration, but also causes damage to multiple organs and tissues (2). Although the pathogenic mechanisms that mediate RA remain to be elucidated, a hallmark of RA pathology is intra-articular inflammation. It has previously been well demonstrated that various active cytokines and chemokines, including TNF- $\alpha$, IL-1 $\beta$, IL- 6 and $\mathrm{C}-\mathrm{X}-\mathrm{C}$ motif chemokine ligand 8/IL-8, are abundant in the arthritic synovium and serum of patients with RA (23), which may impact disease progression leading to articular deterioration and the co-morbidities of RA $(24,25)$. Furthermore, FLS, the resident cells of synovial joints, are associated with the formation of pannus and stimulate inflammatory responses (26). The interaction between inflammatory/immune cell infiltration and FLS is responsible for the progression of joint damage progression and immune activation. Thus, in addition to inhibiting inflammatory responses, targeting FLS may be another important treatment strategy for RA (27).

Targeted therapies have been previously developed and achieved substantial success. For example, TNF- $\alpha$ competitive inhibitor, TNF- $\alpha$ monoclonal antibodies and B-cell-depleting therapies have been used to treat patients with RA $(28,29)$. However, these approaches are usually expensive and have potential side-effects (30). Additionally, traditional medications cannot repair joint damage (31), and most patients develop clinical relapse and disease progression. Thus, it is critical to develop novel and effective therapeutic approaches to improve the clinical outcomes. MSCs have previously been demonstrated to be effective for the treatment of various diseases, including autoimmune arthritis (32), due to their self-regenerating, differentiation and immunosuppressive capability. MSCs are also present in the synovium where they are thought to maintain tissues and contribute to repair processes (33). However, MSCs from patients with RA exhibit lower clonogenic potential and proliferative capability compared with normal MSCs (34), making allogenic MSCs accessible for clinical trials. Furthermore, although bone marrow has been considered as the main source of MSCs, BM-MSCs are not always a viable option for clinical use. Compared with BM-MSCs, UC-MSCs have higher proliferative effectiveness, more robust differentiation ability, and lower risks of contamination and immunogenicity (18). Thus, UC-MSCs demonstrate greater therapeutic potential for the treatment of a number of diseases, compared with BM-MSCs. However, the effect of co-culture of FLS with UC-MSCs on RA has not been widely investigated.

The present study isolated and cultured FLS and UC-MSCs. While the stroma of the synovium includes FLS and MSCs, their association is poorly understood. Therefore, the phenotype of UC-MSCs was subsequently identified. The data of the current study demonstrated that UC-MSCs had been successfully isolated and expanded. The cells were negative for CD14, CD34 and CD45, but positive for CD105, CD29, CD44 and CD166, demonstrating that UC-MSCs exhibited a fibroblast-like morphology. FLS and MSCs may be from the same lineage but at different functional stages. Following identification of the UC-MSC phenotype, FLS and UC-MSCs were co-cultured to 
A

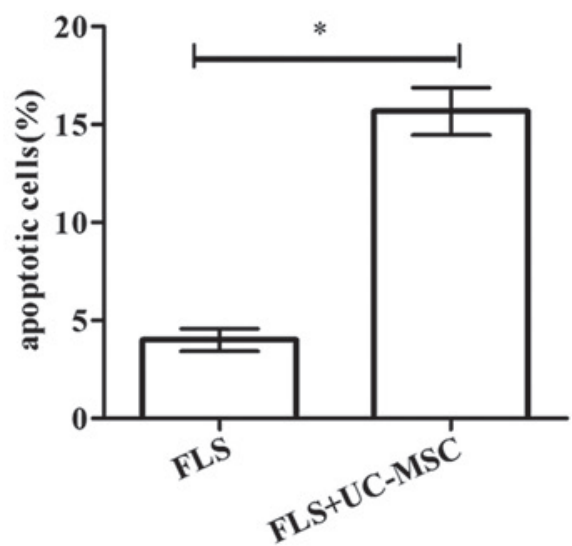

B

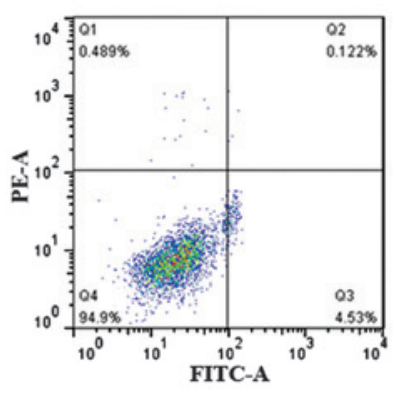

FLS

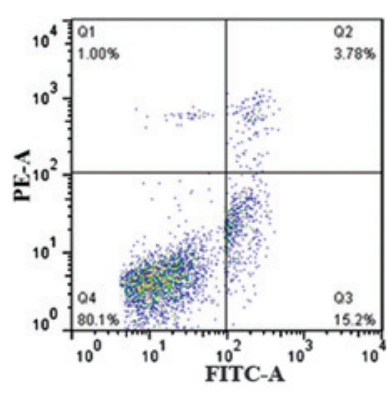

FLS+UC-MSC

Figure 2. Effect of co-culture on apoptosis. (A) Percentage of apoptotic cells. "P $<0.05$, comparison indicated by brackets. (B) Representative images of flow cytometry. FLS, fibroblast-like synoviocyte; UC-MSC, umbilical cord mesenchymal stem cell; PE, phycoerythrin; FITC, fluorescein isothiocyanate.

A

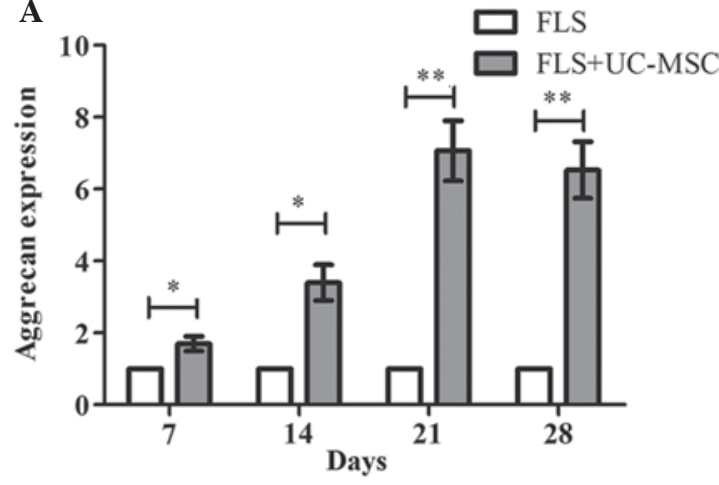

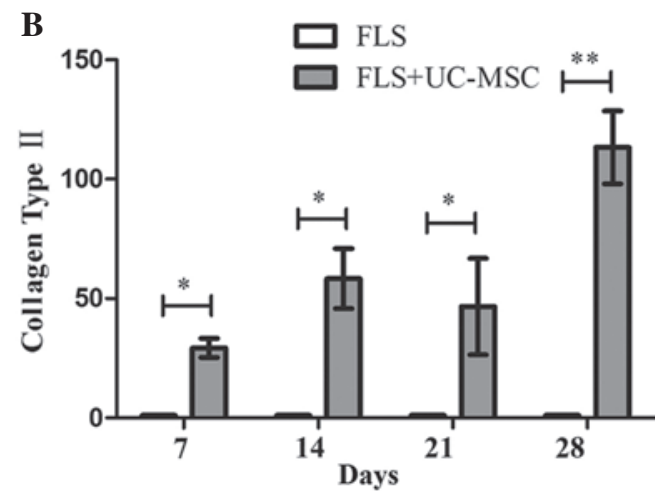

Figure 3. Effect of co-culture on chondrogenesis. (A) Relative expression of aggrecan. (B) Relative expression of collagen type II. ${ }^{*} \mathrm{P}<0.05$, ${ }^{* *} \mathrm{P}<0.01$, comparison indicated by brackets. Data are presented as the mean \pm standard deviation. FLS, fibroblast-like synoviocyte; UC-MSC, umbilical cord mesenchymal stem cells.

A

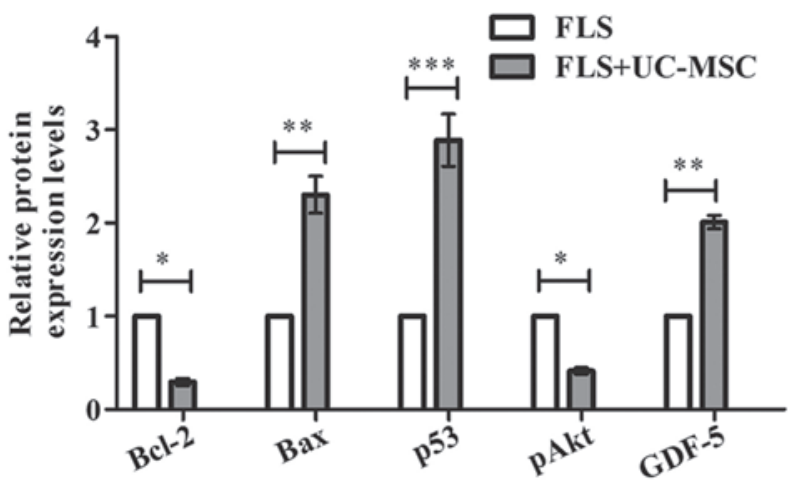

B

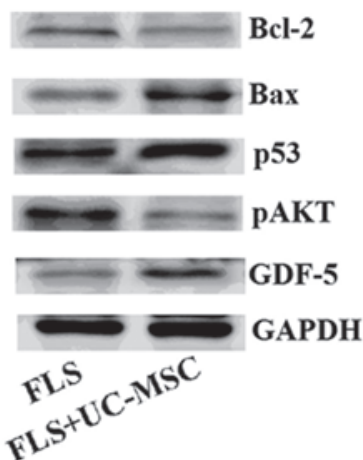

Figure 4. Effect of co-culture on expression of apoptosis-associated proteins. (A) Relative protein expression levels were measured following (B) western blotting. ${ }^{*} \mathrm{P}<0.05,{ }^{* *} \mathrm{P}<0.01,{ }^{* * * *} \mathrm{P}=0.001$, comparison indicated by brackets. Data are presented as the mean \pm standard deviation. FLS, fibroblast-like synoviocyte; UC-MSC, umbilical cord mesenchymal stem cell; Bcl-2, Bcl-2 apoptosis regulator; Bax, Bcl-2-associated X protein; pAKT, phospho-AKT serine/threonine kinase; GDF-5, growth differentiation factor-5.

observe the effect on the expression of pro-inflammatory cytokines and chemokines, and on apoptosis and chondrogenesis. The results of the present study demonstrated that co-culture of FLS with UC-MSCs significantly reduces the expression of IL-1 $\beta$, IL-6 and CCL-2. Among the pro-inflammatory cytokines, IL-1 $\beta$ has previously been reported strongly inhibit tissue repair during RA (35). IL-6 and CCL-2 are also thought to be important in the pathology of RA. Additionally, the current study demonstrated that co-culture of FLS with UC-MSCs significantly increased FLS apoptosis and promoted chondrogenesis. Furthermore, the 
mechanisms that mediated the increases apoptosis and chondrogenesis were explored. The results demonstrated that the levels of anti-apoptotic protein level (Bcl-2 and p-Akt) were significantly decreased, whereas, pro-apoptotic proteins (p53 and Bax) were significantly increased by co-culture of FLS with UC-MSCs, suggesting that the increased apoptosis may be induced via regulation of the expression of pro/anti-apoptotic proteins. The present study also demonstrated that the protein expression level of GDF-5 was significantly increased by co-culture of FLS with UC-MSCs. GDF-5, a member of bone morphogenetic protein and transforming growth factor- $\beta$ families, is expressed by fibroblasts, articular cartilage chondrocytes and odontoblasts (36-38). It was previously reported that GDF-5 aggregates the mesenchyme, increases glycosaminoglycan synthesis, promotes chondrogenic differentiation in vitro, and induces cartilage and bone formation in vivo $(37,39)$. A previous report suggested that GDF-5 is present in the synovium membrane and cartilage of patients with RA, and is actively involved in the regulation of cartilage maintenance and repair (40). The present study demonstrated that GDF-5 may be involved in chondrogenesis induced by co-culture of FLS with UC-MSCs.

In conclusion, co-culture of FLS with UC-MSCs exerts an inhibitory effect on the expression of pro-inflammatory cytokines and chemokines, induces FLS apoptosis and promotes chondrogenesis. The results of the current study suggest that co-culture of FLS with UC-MSCs may be clinically useful and potentially important for the treatment of RA.

\section{References}

1. Scott DL, Wolfe F and Huizinga TW: Rheumatoid arthritis Lancet 376: 1094-1108, 2010.

2. McInnes IB and Schett G: The pathogenesis of rheumatoid arthritis. New Engl J Med 365: 2205-2219, 2011.

3. Smolen JS, Emery P, Fleischmann R, van Vollenhoven RF Pavelka K, Durez P, Guérette B, Kupper H, Redden L, Arora V and Kavanaugh A: Adjustment of therapy in rheumatoid arthritis on the basis of achievement of stable low disease activity with adalimumab plus methotrexate or methotrexate alone: The randomised controlled OPTIMA trial. Lancet 383: 321-332, 2014.

4. Sangha O: Epidemiology of rheumatic diseases. Rheumatology (Oxford) 39 (Suppl 2): 3-12, 2000.

5. Li R, Sun J, Ren LM, Wang HY, Liu WH, Zhang XW, Chen S, Mu R, He J,Zhao Y, et al: Epidemiology of eight common rheumatic diseases in China: A large-scale cross-sectional survey in Beijing. Rheumatology (Oxford) 51: 721-729, 2012.

6. Cross M, Smith E, Hoy D, Carmona L, Wolfe F, Vos T, Williams B, Gabriel S, Lassere M, Johns N, et al: The global burden of rheumatoid arthritis: Estimates from the global burden of disease 2010 study. Ann Rheum Dis 73: 1316-1322, 2014.

7. Uhlig T, Moe RH and Kvien TK: The burden of disease in rheumatoid arthritis. Pharmacoeconomics 32: 841-851, 2014.

8. Hyrich KL, Symmons DP, Watson KD and Silman AJ; British Society for Rheumatology Biologics Register: Comparison of the response to infliximab or etanercept monotherapy with the response to cotherapy with methotrexate or another disease-modifying antirheumatic drug in patients with rheumatoid arthritis: Results from the British Society for Rheumatology Biologics Register. Arthritis Rheum 54: 1786-1794, 2006.

9. Listing J, Strangfeld A, Rau R, Kekow J, Gromnica-Ihle E, Klopsch T, Demary W, Burmester GR and Zink A: Clinical and functional remission: Even though biologics are superior to conventional DMARDs overall success rates remain low--results from RABBIT, the German biologics register. Arthritis Res Ther 8: R66, 2006.

10. Gonzalez-Rey E, Gonzalez MA, Varela N, O'Valle F, Hernandez-Cortes P, Rico L, Büscher D and Delgado M: Human adipose-derived mesenchymal stem cells reduce inflammatory and $\mathrm{T}$ cell responses and induce regulatory $\mathrm{T}$ cells in vitro in rheumatoid arthritis. Ann Rheum Dis 69: 241-248, 2010.
11. Sun L, Akiyama K, Zhang H, Yamaza T, Hou Y, Zhao S, Xu T, Le A and Shi S: Mesenchymal stem cell transplantation reverses multiorgan dysfunction in systemic lupus erythematosus mice and humans. Stem Cells 27: 1421-1432, 2009.

12. De Bari C: Are mesenchymal stem cells in rheumatoid arthritis the good or bad guys? Arthritis Res Ther 17: 113, 2015.

13. Papadopoulou A, Yiangou M, Athanasiou E, Zogas N, Kaloyannidis P, Batsis I, Fassas A, Anagnostopoulos A and Yannaki E: Mesenchymal stem cells are conditionally therapeutic in preclinical models of rheumatoid arthritis. Ann Rheum Dis 71: 1733-1740, 2012.

14. Stolzing A, Jones E, McGonagle D and Scutt A: Age-related changes in human bone marrow-derived mesenchymal stem cells: Consequences for cell therapies. Mech Ageing Dev 129: 163-173, 2008.

15. Baksh D, Yao R and Tuan RS: Comparison of proliferative and multilineage differentiation potential of human mesenchymal stem cells derived from umbilical cord and bone marrow. Stem Cells 25: 1384-1392, 2007.

16. Weiss ML, Anderson C, Medicetty S, Seshareddy KB, Weiss RJ, VanderWerff I, Troyer D and McIntosh KR: Immune properties of human umbilical cord Wharton's jelly-derived cells. Stem Cells 26: 2865-2874, 2008.

17. Liu Y, Mu R, Wang S, Long L, Liu X, Li R, Sun J, Guo J, Zhang X, Guo J, et al: Therapeutic potential of human umbilical cord mesenchymal stem cells in the treatment of rheumatoid arthritis. Arthritis Res Ther 12: R210, 2010.

18. Lu LL, Liu YJ, Yang SG, Zhao QJ, Wang X, Gong W, Han ZB, $\mathrm{Xu} \mathrm{ZS}, \mathrm{Lu} \mathrm{YX}$, Liu D, et al: Isolation and characterization of human umbilical cord mesenchymal stem cells with hematopoiesis-supportive function and other potentials. Haematologica 91: 1017-1026, 2006.

19. Cao Y, Sun Z, Liao L, Meng Y, Han Q and Zhao RC: Human adipose tissue-derived stem cells differentiate into endothelial cells in vitro and improve postnatal neovascularization in vivo. Biochem Biophys Res Commun 332: 370-379, 2005.

20. Arnett FC, Edworthy SM, Bloch DA, McShane DJ, Fries JF, Cooper NS, Healey LA, Kaplan SR, Liang MH, Luthra HS, et al: The American Rheumatism Association 1987 revised criteria for the classification of rheumatoid arthritis. Arthritis Rheum 31: 315-324, 1988.

21. Cho CS, Cho ML, Min SY, Kim WU, Min DJ, Lee SS, Park SH, Choe J and Kim HY: CD40 engagement on synovial fibroblast up-regulates production of vascular endothelial growth factor. J Immunol 164: 5055-5061, 2000.

22. Livak KJ and Schmittgen TD: Analysis of relative gene expression data using real-time quantitative PCR and the 2(-Delta Delta C(T)) method. Methods 25: 402-408, 2001.

23. Moelants EA, Mortier A, Van Damme J and Proost P: Regulation of TNF- $\alpha$ with a focus on rheumatoid arthritis. Immunol Cell Biol 91: 393-401, 2013.

24. Brennan FM and McInnes IB: Evidence that cytokines play a role in rheumatoid arthritis. J Clin Invest 118: 3537-3545, 2008.

25. Kokkonen H, Söderström I, Rocklöv J, Hallmans G, Lejon K and Rantapää Dahlqvist S: Up-regulation of cytokines and chemokines predates the onset of rheumatoid arthritis. Arthritis Rheum 62: 383-391, 2010.

26. Kasperkovitz PV, Timmer TC, Smeets TJ, Verbeet NL, Tak PP, van Baarsen LG, Baltus B, Huizinga TW, Pieterman E, Fero M, et al: Fibroblast-like synoviocytes derived from patients with rheumatoid arthritis show the imprint of synovial tissue heterogeneity: Evidence of a link between an increased myofibroblast-like phenotype and high-inflammation synovitis. Arthritis Rheum 52: 430-441, 2005.

27. Noss EH and Brenner MB: The role and therapeutic implications of fibroblast-like synoviocytes in inflammation and cartilage erosion in rheumatoid arthritis. Immunol Rev 223: 252-270, 2008

28. Dass S, Vital EM and Emery P: Rituximab: Novel B-cell depletion therapy for the treatment of rheumatoid arthritis. Opin Pharmacother 7: 2559-2570, 2006

29. Colmegna I, Ohata BR and Menard HA: Current understanding of rheumatoid arthritis therapy. Clin Pharmacol Ther 91: 607-620, 2012.

30. Giles JT, Bartlett SJ, Gelber AC, Nanda S, Fontaine K, Ruffing V and Bathon JM: Tumor necrosis factor inhibitor therapy and risk of serious postoperative orthopedic infection in rheumatoid arthritis. Arthritis Rheum 55: 333-337, 2006. 
31. Moreland LW, Schiff MH, Baumgartner SW, Tindall EA, Fleischmann RM, Bulpitt KJ, Weaver AL, Keystone EC, Furst DE, Mease PJ, et al: Etanercept therapy in rheumatoid arthritis. A randomized, controlled trial. Ann Intern Med 130: 478-486, 1999.

32. Augello A, Tasso R, Negrini SM, Cancedda R and Pennesi G: Cell therapy using allogeneic bone marrow mesenchymal stem cells prevents tissue damage in collagen-induced arthritis. Arthritis Rheum 56: 1175-1186, 2007.

33. De Bari C, Dell'Accio F, Karystinou A, Guillot PV, Fisk NM, Jones EA, McGonagle D, Khan IM, Archer CW, Mitsiadis TA, et al: A biomarker-based mathematical model to predict bone-forming potency of human synovial and periosteal mesenchymal stem cells. Arthritis Rheum 58: 240-250, 2008

34. Kastrinaki MC, Sidiropoulos P, Roche S, Ringe J, Lehmann S, Kritikos H, Vlahava VM, Delorme B, Eliopoulos GD, Jorgensen C, et al: Functional, molecular and proteomic characterisation of bone marrow mesenchymal stem cells in rheumatoid arthritis. Ann Rheum Dis 67: 741-749, 2008.

35. Catterall JB, Carrère S, Koshy PJ, Degnan BA, Shingleton WD, Brinckerhoff CE, Rutter J, Cawston TE and Rowan AD: Synergistic induction of matrix metalloproteinase 1 by interleukin-1alpha and oncostatin $\mathrm{M}$ in human chondrocytes involves signal transducer and activator of transcription and activator protein 1 transcription factors via a novel mechanism. Arthritis Rheum 44: 2296-2310, 2001.
36. Hötten G, Neidhardt H, Jacobowsky B and Pohl J: Cloning and expression of recombinant human growth/differentiation factor 5. Biochem Biophys Res Commun 204: 646-652, 1994.

37. Bobacz K, Gruber R, Soleiman A, Graninger WB, Luyten FP and Erlacher L: Cartilage-derived morphogenetic protein-1 and-2 are endogenously expressed in healthy and osteoarthritic human articular chondrocytes and stimulate matrix synthesis. Osteoarthritis Cartilage 10: 394-401, 2002.

38. Morotome Y, Goseki-Sone M, Ishikawa I and Oida S: Gene expression of growth and differentiation factors-5, -6, and -7 in developing bovine tooth at the root forming stage. Biochem Biophys Res Commun 244: 85-90, 1998.

39. Hötten GC, Matsumoto T, Kimura M, Bechtold RF, Kron R, Ohara T, Tanaka H, Satoh Y, Okazaki M, Shirai T, et al: Recombinant human growth/differentiation factor 5 stimulates mesenchyme aggregation and chondrogenesis responsible for the skeletal development of limbs. Growth Factors 13: 65-74, 1996.

40. Liu FL, Lin LH, Sytwu HK and Chang DM: GDF-5 is suppressed by IL-1beta and enhances TGF-beta3-mediated chondrogenic differentiation in human rheumatoid fibroblast-like synoviocytes. Exp Mol Pathol 88: 163-170, 2010 . 